\title{
Molecular diagnosis of spinal muscular atrophy
}

\author{
H Stewart, A Wallace, J McGaughran, R Mountford, H Kingston
}

\begin{abstract}
The frequency of deletions within the survival motor neurone (SMN) and neuronal apoptosis inhibitory protein (NAIP) genes in patients with spinal muscular atrophy (SMA), and the impact of this on the diagnosis and prenatal diagnosis of SMA, were investigated by molecular analysis of stored DNA and retrospective review of case notes. In type I SMA, 16 of 17 cases were homozygously deleted for exons 7 and 8 of SMN, 14 of 17 were homozygously deleted for exon 5 of NAIP, and 13 of 17 were deleted for both. In types II and III SMA, seven of nine cases were deleted for exons 7 and 8 of SMN. Deletions of SMN and NAIP occurred in four of nine cases. With one exception, the deletion genotypes of probands, affected siblings, and terminated fetuses were identical. Molecular studies are replacing conventional investigations for SMA and have a high uptake prenatally.

(Arch Dis Child 1998;78:531-535)
\end{abstract}

Keywords: spinal muscular atrophy; survival motor neurone gene; neuronal apoptosis inhibitory protein gene; molecular diagnosis

The spinal muscular atrophies (SMAs) form a heterogeneous group of diseases inherited as autosomal dominant, autosomal recessive, or $\mathrm{X}$ linked recessive traits. ${ }^{12}$ The childhood onset autosomal recessive form affects between one in 6000 and one in 10000 live births and is the second most common lethal autosomal recessive disorder in white people after cystic fibrosis. The carrier frequency is one in $60-80$ in the UK. ${ }^{3}$

The characteristic pathology of SMA is degeneration of motor neurones in the anterior horn of the spinal cord and, in some cases, motor nuclei in the brain stem, resulting in muscular atrophy and progressive paralysis. ${ }^{1}$ Recent studies have advanced the understanding of the possible mechanisms involved in the pathogenesis of SMA. A novel protein (SMN) is located in the cytoplasm and in novel nuclear bodies called gems. SMN is found at high concentrations in motor neurones and the spinal cord in normal subjects but at reduced concentrations in patients with SMA. ${ }^{4} \mathrm{SMN}$ is associated with another novel protein (SIP1).These proteins are thought to play a role in the metabolism of small nuclear RNAs. However, their role in motor neurone degeneration remains undetermined..$^{56}$

There are three clinical subtypes of autosomal recessive SMA. Type I (WerdnigHoffmann disease) is the most severe, with onset in utero, with reduced fetal movements, or in the first six months of life, with hypotonia, wasting, fasiculation, paucity of movement, and areflexia. Affected children have normal intelligence but are unable to sit unaided, have feeding difficulties, and die from respiratory failure or aspiration before the age of 2 years. Type II is of intermediate severity. Patients have hypotonia, delayed motor milestones, are able to sit unsupported, but cannot stand or walk unaided. Complications include scoliosis and feeding difficulties. Survival depends on the degree of respiratory muscle involvement but is usually greater than 4 years. Type III (Kugelberg-Welander disease) has an onset after the age of two and patients are able to walk unaided, although they do so late, fall frequently, and have a shuffling gait, foot drop, and difficulty with stairs. Slow deterioration results in scoliosis and wheel chair dependence.

The diagnosis is confirmed by muscle biopsy (showing degeneration of muscle fibres, in the absence of inflammation, fibrosis, or histochemical abnormality) and electromyelography (EMG) (showing denervation, paucity of movement, and fasciculation). These procedures are invasive, time consuming, and may give inconclusive results when performed in young infants. Findings in cerebrospinal fluid, serum enzymes, and nerve conduction are normal.

In $1990,95 \%$ of cases of all three recessive forms of SMA were found to be linked to the $5 q 13$ region of chromosome $5 .^{78}$ This enabled prenatal prediction on the basis of linkage in some families. ${ }^{910}$ Deletion events within this region were found to be associated with SMA, ${ }^{11}{ }^{12}$ and in 1995, two candidate genes within this region were postulated: the survival motor neurone (SMN) and neuronal apoptosis inhibitory protein (NAIP) genes. ${ }^{13-16}$ This region of the genome is complex and inherently unstable, with two almost identical copies of SMN, one centromeric and one telomeric, and multiple copies of pseudogenes of NAIP ${ }^{17}$ (fig 1).

Homozygous deletions of exons 7 and 8 of the telomeric (functional) copy of SMN occur in $>95 \%$ of patients with SMA but not in normal control populations. ${ }^{18}$ Short deletions in the consensus splice sites of introns 6 and 7,

Centromeric copy

Telomeric copy

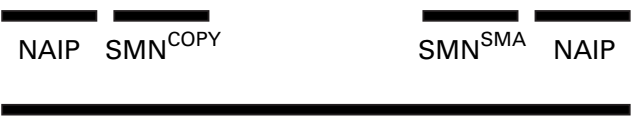

Figure 1 Map of chromosome 5q13. 
Table 1 Results of DNA deletion analysis

\begin{tabular}{llll}
\hline & Type I & Types II and III & All types \\
\hline SMN exons 7 and 8 & $16 / 17(94 \%)^{\star}$ & $7 / 9(78 \%)$ & $23 / 26(88.5 \%)$ \\
NAIP exon 5 & $14 / 17(82 \%)$ & $4 / 9(44 \%)$ & $18 / 26(69 \%)$ \\
Both & $13 / 17(76 \%)$ & $4 / 9(44 \%)$ & $17 / 26(65 \%)$ \\
\hline
\end{tabular}

^Remaining patient was deleted for exon 8 of SMN and exon 5 of NAIP.
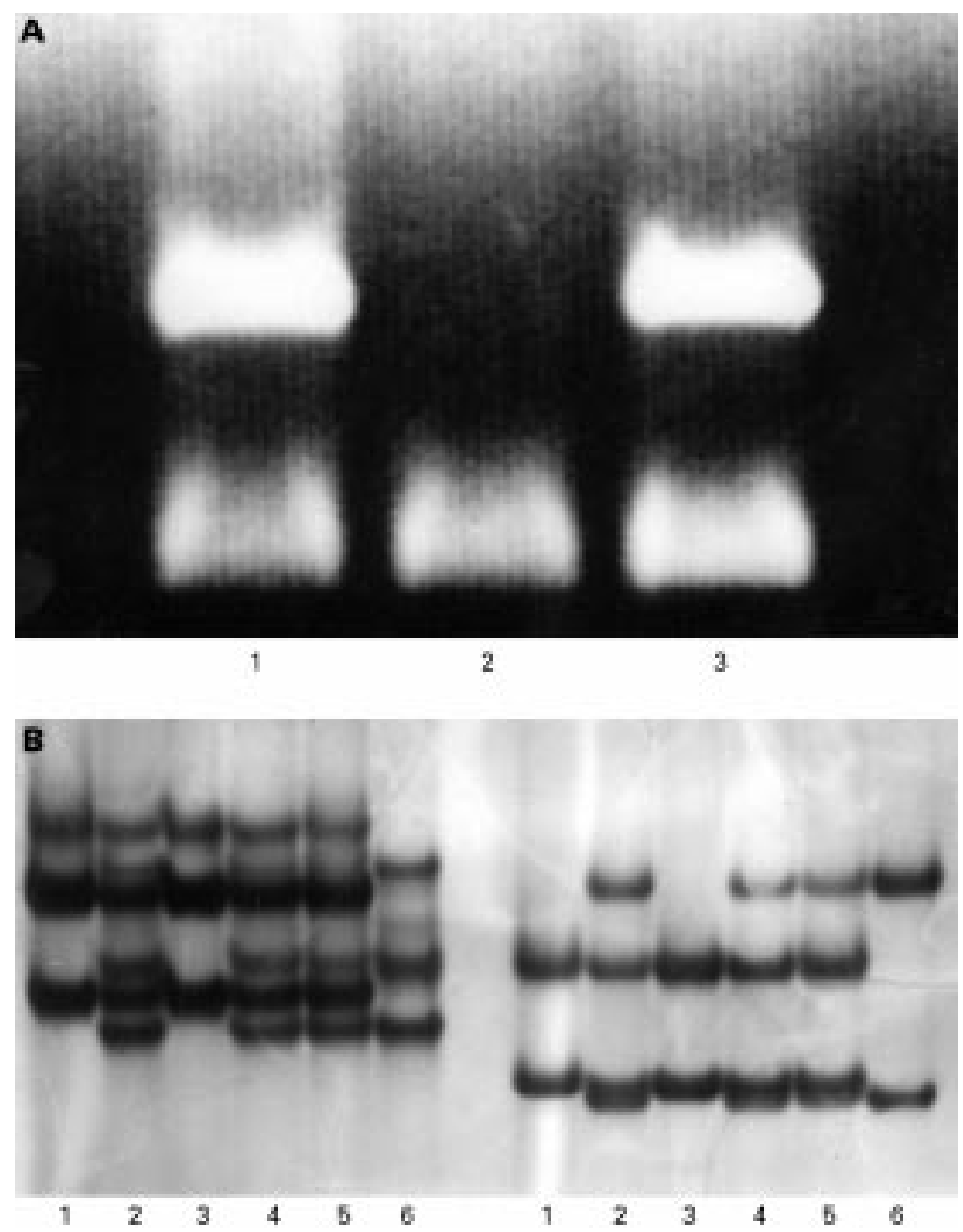

Figure 2 (A) Agarose gel showing polymerase chain reaction (PCR) analysis of neuronal apoptosis inhibitory protein (NAIP) genes. Lane 1, normal control, both exons 5 and 13 amplify. Lanes 2 and 3, both patient samples. Lane 2, homozygous deletion of exon 5 , normal exon 13 supporting a diagnosis of spinal muscular atrophy (SMA); lane 3, no deletion of exon 5. (B) Single strand conformation dependent polymorphism analysis (SSCP) analysis of survival motor neurone (SMN) exons 7 and 8. Lane 1, control sample homozygously deleted for SMN functional gene; lane 2, normal control; lane 3, patient homozygously deleted for exons 7 and 8 of the functional SMN gene, confirming the diagnosis of SMA; lanes 4 and 5, normal pattern, excluding the diagnosis of SMA; lane 6, control sample homozygously deleted for the non-functional SMN gene.

and point mutations, have been found in affected individuals who do not have homozygous deletions of exons 7 and 8 of SMN, confirming that SMN has a role in the aetiology of SMA. ${ }^{16}$ The exact role of deletions of SMN is not clear, as illustrated by the following observations: unaffected parents and siblings have homozygous deletions of exons 7 and 8 of SMN in $1 \%$ of cases $^{20}$; parents of children with types II and III SMA may have more than two copies of the centromeric copy of SMN and it has been postulated that this might have an effect on the phenotype ${ }^{21} ; 4 \%$ of normal people are homozygously deleted for the centromeric copy of SMN, so it is not known whether this copy of the gene has any function. ${ }^{17}{ }^{18}$ Deletions of exon 5 of NAIP occur in $67 \%$ of patients with type I SMA, $42 \%$ of those with types II and III, and $2-3 \%$ of controls. ${ }^{14}$ The role of this gene in the aetiology of SMA remains obscure, although it is thought that larger deletions involving SMN and NAIP are associated with increased disease severity. ${ }^{22}{ }^{23}$ Deletion analysis of SMN has been used for prenatal diagnosis and has largely replaced linkage analysis. As the deletion in the proband arises de novo in $1 \%$ of patients with SMA, errors could be made in prenatal predictions based only on linkage analysis in subsequent pregnancies. ${ }^{24-26}$

The purpose of our study was to confirm the frequency of deletions within SMN and NAIP in patients with known SMA, to establish the pattern of deletions in affected siblings, to confirm that fetuses terminated on the basis of linkage had deletions within these genes, and to review how this information can aid patients and clinicians in diagnosing SMA prenatally and in childhood.

\section{Methods}

SUBJECTS

We included in our study all patients referred to our department before August 1996, in whom the diagnosis of SMA was confirmed clinically, their affected siblings, and those fetuses terminated following prenatal diagnosis. The patients came from a variety of different ethnic origins.

MOLECULAR ANALYSIS

DNA was extracted from peripheral blood lymphocytes on an Applied Biosystems 380A DNA extractor or from stored Guthrie cards by cutting a $3 \mathrm{~mm}^{2}$ fragment of card with a sterile scalpel, boiling the fragment in $100 \mu \mathrm{l}$ of $50 \mathrm{mM}$ sodium hydroxide for 20 minutes, then neutralising with $15 \mu \mathrm{l}$ of $1.0 \mathrm{M}$ Tris, $\mathrm{pH} 8.0 .^{27}$

Polymerase chain reaction (PCR) amplification of SMN exons 7 and 8, and NAIP exons 5 and 13 was carried out in $10 \mu \mathrm{l}$ volumes using $25 \mathrm{ng}$ of genomic DNA, 5 pmol each of forward and reverse primers, $750 \mu \mathrm{mol} / 1$ of each dNTP, $0.2 \mathrm{U}$ Taq polymerase (Gibco BRL, Paisley, UK) in a PCR buffer comprising $3.7 \mathrm{mM} \mathrm{MgCl}_{2}$. PCR was carried out on a Techne PHC-2 using the following cycling parameters: initial denaturation at $94^{\circ} \mathrm{C}$ for three minutes; 30 cycles of $94^{\circ} \mathrm{C}$ for one minute, $60^{\circ} \mathrm{C}$ for one minute, and $72^{\circ} \mathrm{C}$ for one minute; and a final synthesis of $72^{\circ} \mathrm{C}$ for 10 minutes. Owing to the low yield of DNA from Guthrie cards, PCR was extended to 35 cycles. PCR products from NAIP were loaded on to $2 \%$ agarose gels stained with ethidium bromide, run in $1 \times$ Tris borate EDTA (TBE) buffer and visualised under UV light. Exon 13 of NAIP was analysed as a control.

Single strand conformation dependent polymorphism analysis (SSCP) was used to investigate exons 7 and 8 of SMN. SSCP is a simple, sensitive technique in which DNA is amplified by PCR then samples are denatured and loaded on to a non-denaturing polyacrylamide gel. Single stranded DNA has a tendency to fold to form complex structures with different electrophoretic properties depending on their 
Table 2 Outcome of conventional investigations

\begin{tabular}{llllll}
\hline & \multicolumn{2}{l}{ Type I } & & \multicolumn{2}{l}{ Types II and III } \\
\cline { 2 - 3 } \cline { 5 - 6 } & $\begin{array}{l}\text { Biopsy } \\
(n=11)\end{array}$ & $\begin{array}{l}\text { EMG } \\
(n=13)\end{array}$ & & $\begin{array}{l}\text { Biopsy } \\
(n=10)\end{array}$ & $\begin{array}{l}\text { EMG } \\
(n=9)\end{array}$ \\
\hline Diagnostic & 7 & 9 & 9 & 6 \\
Atypical, but compatible & 4 & 1 & & & 0 \\
Atypical & 0 & 2 & & 1 & 0 \\
Normal & 0 & 1 & & & 3
\end{tabular}

Table 3 Outcome of pregnancies

\begin{tabular}{lclll}
\hline & $\begin{array}{l}\text { Number of } \\
\text { pregnancies }\end{array}$ & $\begin{array}{l}\text { Number of } \\
\text { tests }\end{array}$ & $\begin{array}{l}\text { Number of } \\
\text { affected children } \\
\text { born }\end{array}$ & $\begin{array}{l}\text { Number of } \\
\text { terminations }\end{array}$ \\
\hline Type I $(n=21)$ & 3 & 0 & 2 & 0 \\
Prelinkage era $(-1990)$ & 10 & $7^{\star}$ & $3 \dagger$ & $3^{\star}$ \\
Linkage era(1990-95) & 8 & 8 & 0 & 1 \\
Deletion era (1995-) & & & & \\
Types II and III $(n=13)$ & 8 & 0 & 2 & 0 \\
Prelinkage era $(-1990)$ & 5 & $1^{\star}$ & $1 \dagger$ & 0 \\
Linkage era $(1990-95)$ & 0 & 0 & 0 & 0 \\
Deletion era $(1995-)$ & 0 & & & \\
\hline
\end{tabular}

${ }^{\star}$ Linkage results subsequently confirmed by deletion testing; †no prenatal test performed.

conformation, which in turn depends on the DNA sequence. In this case, deleted and normal exons 7 and 8 of the telomeric and centromeric copies of SMN have different mobilities and are, therefore, easily distinguished in affected patients and controls. PCR products were run on $8 \%$ polyacrylamide gels in $10 \times \mathrm{TBE}$ buffer then visualised by silver staining. ${ }^{28}$

Case notes were reviewed retrospectively.

\section{Results}

TYPE I

There were 17 probands, six affected siblings, and four terminated fetuses. Of the 23 children, 11 were girls and 12 were boys. There was consanguinity in five of 17 families. The dates of birth of probands ranged from 1977 to 1995. The age of onset was stated in 16 children and ranged from birth to 6 months, with a mean of 2.4 months. The age at diagnosis was known in 16 children and ranged from 1 month to 7 months, with a mean of 4.1 months. In one case, where parents declined invasive investigations in 1989, the diagnosis was not confirmed until 1996, when a DNA test was performed on a stored neonatal blood spot. All 23 children died at 2 to 16 months, mean age 8.3 months.

Table 1 and fig 2 show the results of SMN and NAIP deletion testing in the 17 probands. In six families there was an affected sibling. Three were tested and found to have the same deletion genotype as their affected siblings. In two families, three pregnancies were terminated following prenatal prediction on the basis of linkage analysis. Subsequently, the fetuses were shown to have the same deletions as their affected siblings. Prenatal deletion testing identified a further fetus who had the same deletion as the affected sibling.

During life, 14 of 23 children had muscle biopsy and/or EMG performed. In three cases, neither investigation was performed: in one case, a previous sibling had a confirmed diagnosis; in one case, parents declined investigations because they were too invasive; and in another case, the baby was born after SMN deletion testing had become possible and DNA analysis alone confirmed the clinical diagnosis. In six cases it was not clear which investigations were performed. The outcomes of these investigations are summarised in table 2 .

After diagnosis in the proband, there were no further pregnancies in five families. Three of these had older, healthy children and two were recent cases (1993 and 1995). The pregnancies occurring in the remaining 12 families are summarised in table 3.

\section{TYPES II AND III}

There were 12 patients (six boys and six girls) from nine families. The parents in two families were consanguineous. The dates of birth ranged from 1969 to 1995 . The mean age of onset was 12.1 months (range 7-24 months) and the mean age at diagnosis was 24.8 months (range 9-48 months). Two patients died at 9 years 5 months and 4 years 11 months.

Table 1 shows the results of SMN and NAIP deletion testing. In the two patients without deletions, the clinical diagnosis was confirmed by muscle biopsy and EMG findings. One additional patient, who was not included in this study, had late onset SMA confirmed by muscle biopsy and EMG and was found to have deletions of exons 7 and 8 of SMN on one chromosome 5 and a gene conversion event (involving a $\mathrm{C}$ to $\mathrm{T}$ transition at nucleotide 873 but not nucleotide 868-44) in exon 7 of the telomeric copy of SMN on the other chromosome 5 . This transition occurred at a site critical for correct splicing of exon 7 , and rendered the gene non-functional. Gene conversion events in exon 7 have been reported previously. ${ }^{19}$

There were three affected siblings from two families. All three were shown to have the same SMN deletion as the index cases. In one case, the proband was homozygously deleted for NAIP but the sibling was not, owing to recombination. No fetuses were terminated.

Muscle biopsy and/or EMG was performed in 11 of 12 cases and results are shown in table 2 . One case was diagnosed by DNA analysis alone.

Three families had no further children after diagnosis of the proband. The pregnancies occurring in the remaining six families are summarised in table 3.

\section{Discussion}

The genetics of autosomal recessive SMA are complicated and the correlation between genotype and phenotype remains obscure. ${ }^{41-23}$ Despite this, in general, identification of homozygous deletions of exons 7 and 8 of SMN in a child with symptoms can be considered diagnostic and can avoid the need for invasive muscle biopsy and EMG testing. ${ }^{26}$

Our series of patients included children diagnosed before DNA tests were available and in one case analysis was performed on DNA extracted with parental consent from a stored neonatal blood spot card. In 12 cases, blood samples had been taken during life and DNA 
stored in anticipation of future tests becoming available.

We observed an overall frequency of $88.5 \%$ for deletions within the SMN gene compared with $95 \%$ in previous reports. Of 17 probands with type I SMA, 16 were homozygously deleted for exons 7 and 8 of SMN and the remaining patient was deleted for exon 8 only. Of the nine probands with type II and III SMA, seven had homozygous deletions of exons 7 and 8 of SMN. The frequency of deletions within the NAIP gene was $82 \%$ in type I and $44 \%$ in types II and III compared with previously reported frequencies of $67 \%$ and $42 \%$, respectively. ${ }^{14}$ Discrepancies between observed and reported figures may be a result of our small sample size. The sensitivity of DNA deletion tests in confirming the diagnosis of SMA compares favourably with that of muscle biopsy (16 of 21) and EMG (15 of 22). Of four cases of SMA in our series diagnosed since 1995, only one has undergone a muscle biopsy; one was diagnosed on EMG and DNA analysis and two have been diagnosed on DNA analysis alone. The specificity of DNA testing is reflected in reported figures of $<1 \%$ of unaffected relatives with homozygous deletions of SMN and $2-3 \%$ of non-SMN chromosomes having deletions of NAIP..$^{14} 18$

DNA analysis is particularly useful in confirming the diagnosis of SMA in cases with atypical clinical features or atypical results on muscle biopsy or EMG. Severe neonatal SMA with diaphragmatic paralysis has been reported in two siblings discordant for DNA markers flanking the SMA locus, suggesting that this form of SMA maps outside the 5q11.2-q13.3 region. ${ }^{29}$ In our series of patients, there were two babies with neonatal onset of severe SMA with early respiratory problems who died at 8 and 10 weeks. The muscle biopsy in both was not typical of SMA, but was thought to be compatible with the diagnosis when the early timing of the investigations was taken into account. Both patients had EMGs showing active denervation, consistent with SMA. Subsequent analysis of DNA in both cases revealed homozygous deletions of exons 7 and 8 of SMN, confirming the diagnosis and the validity of a prenatal test that had been performed by linkage analysis in one of the families. Both patients were also homozygously deleted for exon 5 of NAIP, which is compatible with their severe phenotype.

In general, the limitations of linkage analysis as a result of non-informativeness or recombination can be resolved by the more direct deletion testing. ${ }^{1125}$ In our series, two children at risk of SMA type II had predictive tests performed by DNA linkage analysis during the first 6 months of life. In both cases prenatal testing had been declined. The first child was calculated to be at $3.5 \%$ risk of being affected. Later, he developed symptoms of SMA and the diagnosis was confirmed by open muscle biopsy at 13 months of age. Further linkage analysis using closer markers confirmed that the affected child had inherited the same SMA linked haplotyes as the older affected sibling, indicating that a recombination had occurred

\section{Key messages}

- All patients should be clinically assessed by a paediatric neurologist.

- DNA should be analysed in all cases of suspected spinal muscular atrophy.

- Counselling should be offered as well as prenatal diagnosis via linkage and deletion testing.

- Samples should be stored for future mutation testing.

between the disease locus and the original DNA markers used. Subsequent analysis confirmed homozygous deletion of exons 7 and 8 of SMN in both siblings. In the second family, the child was tested after birth and found to have inherited the same high risk chromosomes as her affected sibling. Unlike her sibling, however, she was not homozygously deleted for exons 7 and 8 of SMN. It was concluded that only one parent was a carrier for SMA and that the other parent had germinal mosaicism for the deletion or that an additional de novo deletion had arisen in the affected child.

In our series, there was a high uptake of prenatal prediction by linkage alone (eight of 15 pregnancies) and of prenatal diagnosis by both deletion testing and linkage (eight of eight pregnancies). This reflects the high level of acceptability of prenatal diagnosis and termination of affected pregnancy reported previously. ${ }^{10}$

The pattern of SMN and NAIP deletions in affected siblings and terminated fetuses was identical to that in the proband except in one case where a recombination was known to have occurred, resulting in deletion of SMN but not NAIP in the second affected sibling. Previously, consistency of the deletion has been assumed for SMN deletions but not documented. ${ }^{25}$ Conversely, there are reports of siblings with identical SMN deletions who are not concordant for symptoms of SMA (types II/III)..$^{21}$ This is sufficiently rare that the demonstration of a homozygous deletion of SMN in the presence of symptoms of SMA can be assumed to confirm the diagnosis. In the context of prenatal diagnosis, it is not possible to confirm the phenotype of the fetus, and diagnosis relies solely on determining the presence or absence of the mutation known to cause SMA in the affected sibling. In our series, all fetuses predicted to be affected by linkage analysis have had the presence of homozygous deletions confirmed by subsequent analysis. All fetuses predicted to be unaffected by prenatal linkage or deletion testing have remained healthy after birth.

We thank Drs M Clarke, M Noronha, and P Tomlin for the clinical evaluation and diagnosis of the patients in this study and for referring them for genetic assessment. The muscle biopsies were reported by Drs A Kelsey and P Lynch. Financial support for this study was provided through an Innovative Development for this study was provided through an Innovati
Grant from the NHS Executive North West.

1 Zerres K, Rudnik-Schoneborn S. Spinal muscular atrophies. In: Rimoin DL, Connor JM, Pyeritz RE, eds. Principles and practice of medical genetics. New York: Churchill Livingstone, 1997:2387-403. 
2 Baraitser M. Anterior horn cell disease. In: Motulsky AG, Bobrow M, Harper PS, Scriver C, eds. The genetics of neurological disorders. Oxford: Oxford University Press, 1990:248-66.

3 Pearn J. The gene frequency of acute Werdnig-Hoffmann disease (SMA type I). A total population survey in north east England. $\mathcal{F}$ Med Genet 1973;10:260-5.

4 Coovert D, Le T, McAndrew P, et al. The survival motor neuron protein in spinal muscular atrophy. Hum Mol Gene 1997;6:1205-14

5 Liu Q, Fischer U, Wang F, Dreyfuss G. The spinal muscular atrophy disease gene product, SMN, and its associated protein SIP1 are in a complex with spliceosomal snRNP proteins. Cell 1997;90:1013-21.

6 Fischer U, Liu Q, Dreyfuss G. The SMN-SIP1 complex has an essential role in spliceosomal snRNP biogenesis. Cell 1997;90:1023-9.

7 Brzustowicz LM, Lehner T, Castilla LH, et al. Genetic mapping of chronic childhood-onset spinal muscular atrophy to ping of chronic childhood-onset spinal muscular atroph

8 Melki J, Sheth P, Abdelhak S, et al. Mapping of acute (type I) spinal muscular atrophy to chromosome 5q12-14. LanI) spinal muscular a

9 Daniels RJ, Suthers GK, Morrison KE, et al. Prenatal prediction of spinal muscular atrophy. $\mathcal{F}$ Med Genet 1992;29:165-70

10 Wirth B, Rudnik-Schoneborn S, Hahnen E, Rohrig D, Zerres K. Prenatal prediction in families with autosoma recessive proximal spinal muscular atrophy (5q11.2q13.3): molecular genetics and clinical experience in 109 cases. Prenat Diagn 1995;15:407-17.

11 Melki J, Lefebvre S, Burglen L, et al. De novo and inherited deletions of the $5 \mathrm{q} 13$ region in spinal muscular atrophies. Science 1994;264:1474-7.

12 Daniels RJ, Campbell L, Rodrigues NR, et al. Genomic rearrangements in childhood spinal muscular atrophy: linkage disequilibrium with a null allele. 7 Med Genet 1995 ; 32:93-6.

13 Lewin B. Genes for SMA: multum in parvo. Cell 1995;80:1-5

14 Roy N, Mahadevan MS, McLean M, et al. The gene for neuronal apoptosis inhibitory protein is partially deleted in individuals with spinal muscular atrophy. Cell 1995;80. 167-78.

15 Thompson TG, DiDonato CJ, Simard LR, et al. A novel cDNA detects homozygous microdeletions in greater than $50 \%$ of type I spinal muscular atrophy patients. Nat Genet 1995;9:56-62.
16 Lefebvre S, Burglen L, Reboullet S, et al. Identification and characterization of a spinal muscular atrophy-determining Cell 1995;80:155-65.

17 Morrison KE, Qureshi SJ, Anderson S, et al. Novel ranscribed sequences represented in the complex genomic region 5q13. Biochim Biophys Acta 1996;1308:97-102.

18 Rodrigues NR, Owen N, Talbot K, et al. Deletions in the survival motor neuron gene on $5 \mathrm{q} 13$ in autosomal recessive spinal muscular atrophy. Hum Mol Genet 1995;4:631-4.

19 Bussaglia E, Clermont O, Tizzano E, et al. A frame-shift deletion in the survival motor neurone gene in Spanish spinal muscular atrophy patients. Nat Genet 1995;11:335-7.

20 Wang $\mathrm{CH}, \mathrm{Xu} \mathrm{J}$, Carter TA, et al. Characterization of survival motor neuron $\left(\mathrm{SMN}^{\mathrm{T}}\right)$ gene deletions in asymptosurvival motor neuron (SMN gene deletions in asympto1996;5:359-65.

21 Velasco E, Valero C, Valero A, Moreno F, Hernandez-Chico C. Molecular analysis of the SMN and NAIP genes in Spanish spinal muscular atrophy (SMA) families and correlation between number of copies of ${ }^{\mathrm{C}} \mathrm{BCD} 541$ and SMA phenotype. Hum Mol Genet 1996;5:257-63.

22 Rodrigues NR, Owen N, Talbot $\mathrm{K}$, et al. Gene deletions in spinal muscular atrophy. F Med Genet 1996;33:93-6.

23 Burlet $\mathrm{P}$, Burglen L, Clermont $\mathrm{O}$, et al. Large scale deletions of the $5 \mathrm{q} 13$ region are specific to Werdnig-Hoffmann disease. 7 Med Genet 1996;33:281-3.

24 Capon F, Lo Cicero S, Levato C, Novelli G, Dallapiccola B. De novo deletions of the $5 \mathrm{q} 13$ region and prenatal diagnosis of spinal muscular atrophy. Prenat Diagn 1995;15:93-4.

25 Cobben JM, Scheffer H, De Visser M, et al. Prenatal prediction of spinal muscular atrophy. Experience with linkage studies and consequences of present deletion analysis. Eur f Hum Genet 1996;4:231-6.

26 Cobben JM, van der Steege G, Grootscholten P, de Visser M, Scheffer H, Buys CHCM. Deletions of the survival motor neuron gene in unaffected siblings of patients with spinal muscular atrophy. Am f Hum Genet 1995;57:805-8.

27 Sambrook J, Frisch E, Maniatis T. Molecular cloning: a laboratory manual. 2nd ed. Cold Spring Harbor, New York: Cold Spring Harbor Laboratory, 1989.

28 Orita M, Iwahana H, Kanazawa H, Hayashi K, Sekiya T. Detection of polymorphisms of human DNA by gel electrophoresis as single-strand conformation polymorphisms. Proc Natl Acad Sci USA 1989;86:2766-70.

29 Novelli G, Capon F, Tamisari L, et al. Neonatal spinal muscular atrophy with diaphragmatic paralysis is unlinked to 5q11.2-q13. f Med Genet 1995;32:216-19. 